\title{
WHEN THE ROBIN INEQUALITY DOES NOT HOLD
}

\author{
FRANK VEGA
}

\begin{abstract}
In mathematics, the Riemann Hypothesis is a conjecture that the Riemann zeta function has its zeros only at the negative even integers and complex numbers with real part $\frac{1}{2}$. It is one of the seven Millennium Prize Problems selected by the Clay Mathematics Institute to carry a US 1,000,000 prize for the first correct solution. In 1915, Ramanujan proved that under the assumption of the Riemann Hypothesis, the inequality $\sigma(n)<e^{\gamma} \times n \times \log \log n$ holds for all sufficiently large $n$, where $\sigma(n)$ is the sum-of-divisors function and $\gamma \approx 0.57721$ is the Euler-Mascheroni constant. In 1984, Guy Robin proved that the inequality is true for all $n>5040$ if and only if the Riemann Hypothesis is true. Let $n>5040$ be $n=r \times q$, where $q$ denotes the largest prime factor of $n$. If $n>5040$ is the smallest number such that Robin inequality does not hold, then we show the following inequality is also satisfied: $\sqrt[q]{e}+\frac{\log \log r}{\log \log n}>2$.
\end{abstract}

\section{INTRODUCTION}

As usual $\sigma(n)$ is the sum-of-divisors function of $n$ Cho+07:

$$
\sum_{d \mid n} d
$$

Define $f(n)$ to be $\frac{\sigma(n)}{n}$. Say $\operatorname{Robins}(n)$ holds provided

$$
f(n)<e^{\gamma} \times \log \log n .
$$

The constant $\gamma$ is the Euler-Mascheroni constant, and log is the natural logarithm. The importance of this property is:

Theorem 1.1. [RH] If Robins $(n)$ holds for all $n>5040$, then the Riemann Hypothesis is true [Rob84.

There are several known results about the possible counterexamples of Robins $(n)$ when $n>5040$ Cho+07]. In addition, we show that

2010 Mathematics Subject Classification. Primary 11M26; Secondary 11A41.

Key words and phrases. Riemann hypothesis, Robin inequality, sum-of-divisors function, prime numbers. 
Theorem 1.2. [counterexample] Let $n>5040$ be $n=r \times q$, where $q$ denotes the largest prime factor of $n$. If $n>5040$ is the smallest number such that Robins $(n)$ does not hold, then

$$
\sqrt[q]{e}+\frac{\log \log r}{\log \log n}>2 .
$$

\section{Some Useful Lemmas}

The following lemma is a very helpful inequality:

Lemma 2.1. [ineq] We have

$$
\frac{x}{1-x} \leq \frac{1}{y+y^{2}+\frac{y^{3}}{2}}
$$

where $y=1-x$.

Proof. We know $1+x \leq e^{x}$ Koz21]. Therefore,

$$
\frac{x}{1-x} \leq \frac{e^{x-1}}{1-x}=\frac{1}{(1-x) \times e^{1-x}}=\frac{1}{y \times e^{y}} .
$$

However, for every real number $y \in \mathbb{R}$ Koz21]:

$$
y \times e^{y} \geq y+y^{2}+\frac{y^{3}}{2}
$$

and this can be transformed into

$$
\frac{1}{y \times e^{y}} \leq \frac{1}{y+y^{2}+\frac{y^{3}}{2}} .
$$

Consequently, we show

$$
\frac{x}{1-x} \leq \frac{1}{y+y^{2}+\frac{y^{3}}{2}} .
$$

Here, it is another practical result:

Lemma 2.2. [prop] Suppose that $n>5040$ and let $n=r \times q$, where $q$ denotes the largest prime factor of $n$. We have

$$
f(n) \leq\left(1+\frac{1}{q}\right) \times f(r) .
$$

Proof. Suppose that $n$ is the form of $m \times q^{k}$ where $q \nmid m$ and $m$ and $k$ are natural numbers. We have that

$$
f(n)=f\left(m \times q^{k}\right)=f(m) \times f\left(q^{k}\right)
$$


since $f$ is multiplicative and $m$ and $q$ are coprimes Voj20]. However, we know that

$$
f\left(q^{k}\right) \leq f\left(q^{k-1}\right) \times f(q)
$$

because of we notice that $f(a \times b) \leq f(a) \times f(b)$ when $a, b \geq 2[\operatorname{Voj} 20]$. In this way, we obtain that

$$
f\left(q^{k-1}\right) \times f(q)=f\left(q^{k-1}\right) \times\left(1+\frac{1}{q}\right)
$$

according to the value of $f(q)$ [Voj20]. In addition, we analyze that

$$
f(m) \times f\left(q^{k-1}\right)=f\left(m \times q^{k-1}\right)=f(r)
$$

because $f$ is multiplicative and $m$ and $q$ are coprimes |Voj20]. Finally, we obtain that

$$
f(n)=f(m) \times f\left(q^{k}\right) \leq f(m) \times f\left(q^{k-1}\right) \times f(q)=f(r) \times\left(1+\frac{1}{q}\right)
$$

and as a consequence, the proof is completed.

\section{Proof of Main Theorem}

Theorem 3.1. Let $n>5040$ be $n=r \times q$, where $q$ denotes the largest prime factor of $n$. If $n>5040$ is the smallest number such that $\operatorname{Robins}(n)$ does not hold, then

$$
\sqrt[q]{e}+\frac{\log \log r}{\log \log n}>2 .
$$

Proof. Suppose that $n$ is the smallest integer exceeding 5040 that does not satisfy the Robin's inequality. Let $n=r \times q$, where $q$ denotes the largest prime factor of $n$. In this way, the following inequality

$$
f(n) \geq e^{\gamma} \times \log \log n
$$

should be true. We know that

$$
\left(1+\frac{1}{q}\right) \times f(r) \geq f(q \times r) \geq f(n) \geq e^{\gamma} \times \log \log n
$$

due to lemma 2.2 [prop]. Besides, this shows that

$$
\left(1+\frac{1}{q}\right) \times e^{\gamma} \times \log \log r>e^{\gamma} \times \log \log n
$$

should be true as well. Certainly, if $n$ is the smallest counterexample exceeding 5040 of the Robin's inequality, then Robins $(r)$ holds [Cho+07]. That is the same as

$$
\left(1+\frac{1}{q}\right) \times \log \log r>\log \log n .
$$


We have that

$$
\left(1+\frac{1}{q}\right) \times \log \log r>\log (\log r+\log q)
$$

where we notice that $\log (a+c)=\log a+\log \left(1+\frac{c}{a}\right)$. This follows

$$
\left(1+\frac{1}{q}\right) \times \log \log r>\log \log r+\log \left(1+\frac{\log q}{\log r}\right)
$$

which is equal to

$$
(1+q) \times \log \log r>q \times \log \log r+q \times \log \left(1+\frac{\log q}{\log r}\right)
$$

and thus,

This implies that

$$
\log \log r>q \times \log \left(1+\frac{\log q}{\log r}\right)
$$

$$
\begin{aligned}
\frac{\log \log r}{\log \left(1+\frac{\log q}{\log r}\right)} & = \\
\frac{\log \log r}{\log \frac{\log r+\log q}{\log r}} & = \\
\frac{\log \log r}{\log \frac{\log n}{\log r}} & = \\
\frac{\log \log r}{\log \log n-\log \log r} & = \\
\frac{\log \log r}{\log \log n \times\left(1-\frac{\log \log r}{\log \log n}\right)} & = \\
\frac{\log \log r}{\log \log n} & >q \\
\left(1-\frac{\log \log r}{\log \log n}\right) &
\end{aligned}
$$

should be true. If we assume that $y=1-\frac{\log \log r}{\log \log n}$, then we analyze that

$$
\frac{1}{y+y^{2}+\frac{y^{3}}{2}} \geq \frac{\frac{\log \log r}{\log \log n}}{\left(1-\frac{\log \log r}{\log \log n}\right)}
$$

because of lemma 2.1 [ineq]. As result, we have that

$$
\frac{1}{y+y^{2}+\frac{y^{3}}{2}}>q
$$

and therefore,

$$
\frac{1}{1+y+\frac{y^{2}}{2}}>q \times y .
$$


Since we have

$$
1+y+\frac{y^{2}}{2}>1
$$

then

$$
\frac{1}{1+y+\frac{y^{2}}{2}}<1 .
$$

Consequently, we obtain that

$$
1>q \times y
$$

which is the same as

$$
e>e^{q \times y} .
$$

Because of we have that $1+y \leq e^{y}$ [Koz21], then

$$
e>e^{q \times y} \geq(1+y)^{q}=\left(2-\frac{\log \log r}{\log \log n}\right)^{q}
$$

that is

and finally,

$$
\sqrt[q]{e}>\left(2-\frac{\log \log r}{\log \log n}\right)
$$

$$
\sqrt[q]{e}+\frac{\log \log r}{\log \log n}>2
$$

\section{REFERENCES}

[Cho+07] YoungJu Choie et al. "On Robin's criterion for the Riemann hypothesis". In: Journal de Théorie des Nombres de Bordeaux 19.2 (2007), pp. 357-372. DOI: 10.5802/jtnb.591.

[Koz21] László Kozma. Useful Inequalities. http://www . lkozma . net/inequalities_cheat_sheet/ineq.pdf. Accessed on 2021-09-18. 2021.

[Rob84] Guy Robin. "Grandes valeurs de la fonction somme des diviseurs et hypothèse de Riemann". In: J. Math. pures appl 63.2 (1984), pp. 187-213.

[Voj20] Robert Vojak. "On numbers satisfying Robin's inequality, properties of the next counterexample and improved specific bounds". In: arXiv preprint arXiv:2005.09307 (2020).

CopSonic, 1471 Route de Saint-Nauphary 82000 Montauban, France

E-mail address: vega.frank@gmail.com 